\title{
CENTRAL NERVOUS SYSTEM VASCULITIS IN A PATIENT WITHCROHN'S DISEASE: A CASE REPORT
}

Caio Grafanassi', Fernanda Dornelas Nogueira dos Santos', Gabriel Magevski Garcia', Leticia Scopel Miossi', Carolina Zorzanelli Costa ${ }^{1, \star}$, Raquel Altoé Giovelli', Elizandra Tomazela Laurenti Polito², Ana Paula Santos Louro³, Renata Scarpat Careta ${ }^{4}$, Danilo Manuel Cerqueira Costa ${ }^{5}$, Fabiano Quarto Martins ${ }^{1}$, José Antônio Fiorot Junior', Valquiria Garcia Dinis ${ }^{1}$

1.Escola Superior de Ciências da Saúde da Santa Casa de Misericórdia de Vitória, Vitória (ES), Brazil. 2.Hospital Estadual Dório Silva, Vitória (ES), Brazil. 3.Laboratório PAT, Vitória (ES), Brazil. 4.Universidade Federal do Espírito Santo, Vitória (ES), Brazil. 5.Universidade Federal de São Paulo, São Paulo (SP), Brazil.

*Corresponding author: carolzorzac@gmail.com

\section{BACKGROUND}

Extraintestinal manifestations of inflammatory bowel disease (IBD) occur in 6-40\% of patients and can affect all systems. However, central nervous system (CNS) vasculitis secondary to Crohn disease (CD) is a rare manifestation.

\section{CASE REPORT}

A 34-year-old-man with diagnosis of CD treated with mesalazine $4800 \mathrm{mg} /$ day who had a previous history of fever, oligoarthritis and subungual lesions that resembled cutaneous vasculitis treated with prednisone $20 \mathrm{mg} /$ day with gradual tapper, presented on the medical service reporting a history that, 6 months after prednisone interruption, he developed acute left frontotemporal headache with progressive pain worsening associated with behavior alteration, reduced attention and memory failure. He had sought medical care for several times, but only symptomatic medications had been prescribed. Months after, he evolved with syncope and paresis/ paresthesia on the left body side. He was admitted at the hospital for investigation and exams reveled: liquor with lymphocytic pleocytosis and elevated protein and lgG:6 (VR: 0.3-3.4 mg/dL) levels; tests were normal for glucose level, protein electrophoresis, oligoclonal bands, oncotic cytology. All infections were ruled out after polymerase chain reaction, cultures and serologies liquor analysis. A magnetic resonance (MRI) reveled: T1 hyposignal, T2 and FLAIR hypersignal in subcortical and deep white substance of the left frontal lobes, corpus callosum, putamen, caudate nucleus and bridge associated with marked hyposignal in the gradientecho sequence inferring hematic residues. The conclusion of probable CNS vasculitis. Cerebral angiography revealed occlusion on the right and moderate/severe stenosis on the left anterior cerebral arteries. Central nervous system biopsy showed inflammatory granulomatous infiltrate around the vessels compatible in CD. There were negative/normal tests for ANCA, complement levels, urine test, proteinuria, chest and sinus tomography, ANA, rheumatoid factor. All infectious etiologies were ruled out. Diagnosis of CNS vasculitis related to $\mathrm{CD}$ was made and pulse therapy with methylprednisolone $1 \mathrm{~g} /$ day for 5 days was performed; oral prednisone $1 \mathrm{mg} / \mathrm{kg} /$ day was prescribed with progressive tapper associated with 9 mensal intravenous cyclophosphamide (CFF) (dose: $0.75 \mathrm{~g} / \mathrm{m}^{2}$ ) pulse therapies. The patient currently received $8 \mathrm{CFF}$ doses and had improved in clinical and imaging tests.

\section{CONCLUSION}

Manifestations on CNS occur in IBD and are mostly a consequence of coagulation system alteration or medication complications. Central nervous system vasculitis secondary to CD is rare and only reported in a few cases. Symptoms, laboratory, imaging and biopsy findings are similar to the isolated primary angiitis of the CNS. Intestinal CD activity do not parallel with CNS manifestations making diagnosis challenging. Here, we presented a case of CNS vasculitis related to $C D$ that had a good evolution after combination therapy with glucocorticoid and cyclophosphamide. 